\title{
Determination of the material elasticity modulus in bending
}

\author{
V. Yu. Goltsev, A. V. Osintsev ${ }^{\dagger}$, A. S. Plotnikov \\ †AVOsintsev@yandex.ru
}

National research nuclear university «MEPhI», 31 Kashirskoe shosse, 115409, Moscow, Russia

The beam bend theory allows calculation of material elasticity modulus (EM) based on the ratio of distance between the supports to the height of the sample cross-section $(l / h)$ and the values of specimen points displacements induced by load. We used computational and experimental approaches to define the range of $l / h$ allowing correct EM calculation. In computer simulations performed in ANSYS Mechanical 16.2 at a given EM and at a range of $l / h$ vertical displacements at six points along the external side of the sample in sections under the load and above the support beams were predicted. These displacements were used to re-compute the EM using the formulas of the beam bend theory. Similar measurements were performed in experiment for steel and graphite samples. Specifically, samples were loaded under the scheme of three-point bending and the measurements of the displacements on the surface of the sample were obtained by digital image correlation method. Accordingly, the EM was computed using the beam bend theory method. No relation between the strength characteristics of the investigated materials (as determined during the bending test) and the $l / h$ was found. Yet a good agreement between the EM values obtained by modeling and experimental approach was observed. The effect of $l / h$ on the accuracy of the EM calculation was estimated. Significant correlation between the EM obtained in this study (both by modeling and experiment) and its known values for the examined materials was obtained when $l / h>7$.

Keywords: elastic modulus, three-point bending, the method of digital image correlation, finite element method.

\section{Определение модуля упругости материала при изгибе}

\author{
Гольцев В. Ю., Осинцев А. В. ${ }^{\dagger}$, Плотников А.С. \\ †AVOsintsev@yandex.ru
}

Национальный исследовательский ядерный университет «МИФИ», Каширское шоссе 31, 115409, Москва, Россия

Выполнен расчетный и экспериментальный анализы влияния отношения расстояния между опорами к высоте сечения образца, нагружаемого по схеме трехточечного изгиба, на измеряемый модуль упругости материала. Моделирование производилось в верифицированном комплексе конечно-элементного анализа ANSYS Mechanical версии 16.2. В результате расчетного анализа определены вертикальные перемещения в шести точках внешней боковой поверхности образца в сечениях под нагружающим и над опорными валками. По полученным перемещениям точек поверхности образца восстановлены значения модуля упругости материала. Аналогичные результаты получены при экспериментальном исследовании образцов, изготовленных из стали и графита. Для точного измерения перемещений точек поверхности образца в сечениях под нагружающим и над опорными валками использовали метод цифровой корреляции изображений. По измеренным перемещениям точек поверхности образца определяли модуль упругости материала в зависимости от отношения расстояния между опорами к высоте сечения образца. Отмечено хорошее соответствие результатов расчетного и экспериментального анализов. Выявлены расхождения в оценках перемещений точек в сечениях под нагружающим и над опорными валками в значениях восстановленных модулей упругости. Оценено влияние отношения расстояния между опорами к высоте сечения образца на достоверность определяемого модуля упругости материала. Полученные значения модулей упругости стали и графита хорошо согласуются со значениями, характерными для исследованных материалов, когда отношение расстояния между опорами к высоте сечения образца больше 7. Показано, что прочностные характеристики исследованных материалов, определенные при испытании на изгиб, не зависят от отношения $l / h$.

Ключевые слова: модуль упругости, трехточечный изгиб, метод цифровой корреляции изображений, метод конечных элементов. 


\section{1. Введение}

В настоящее время прогнозирование работы сложных изделий техники в эксплуатации осуществляется путем моделирования условий их нагружения с применением метода конечных элементов [1-3]. Для анализа напряженно деформированного состояния в различных точках изделий необходимо знать механические свойства и константы упругости материалов. Однако не всегда представляются возможности для изготовления стандартных образцов на растяжение, например, по ГОСТ 1497 - 84, или использования резонансного метода при определении модуля упругости материала. Это происходит в случае, когда недостаточно количества произведенного материала или невозможно изготовить образцы требуемой формы. С таким явлением мы сталкиваемся, например, при необходимости изготовления образцов из керамики.

Изготовление образца в виде балки для испытания на изгиб не представляет никакой сложности. Однако всегда возникает вопрос о размере образца, справедливости соотношений его размеров и условий нагружения для удовлетворения требованиям балочной теории, чтобы получать достоверные значения механических свойств материала и модуля Юнга.

Цель работы - исследование влияния размеров образца и условий нагружения на механические свойства и модуль упругости материала. Расчетный анализ осуществлялся методом конечных элементов, и испытывали на трехточечный изгиб прямоугольные пластины с отношением высоты к ширине сечения $h / b=2$. Отношения расстояний между опорами к высоте сечения $l / h$ принимали равным 4, 7 и 10. Перемещение точек образца измеряли с помощью метода цифровой корреляции изображений (ЦКИ).

\section{2. Расчетный анализ влияния условий нагружения образца на измеряемый модуль упругости материала}

Для предварительной оценки влияния соотношений расстояния между опорами нагружаемого образца и высоты его поперечного сечения на достоверность определения характеристик упругости, было проведено математическое моделирование трёхточечного изгиба брусков трёх типоразмеров. Моделирование производилось в верифицированном $[4,5]$ комплексе конечноэлементного анализа ANSYS Mechanical версии 16.2.

Материалы объёмного бруска, нагружающего и опорных валков приняты изотропно-упругими с модулями упругости соответственно 11 и 200 ГПа для графитового и стального брусков и 200 ГПа для стальных валков. Поскольку, такая схема нагружения обладает ярко выраженной симметрией, конечно-элементная модель представляла собой $1 / 4$ часть бруска и валков.

На плоскостях симметрии задавались граничные условия симметрии, которые в данном случае представляют собой запрет перемещений узлов из этих плоскостей. На нижней половине цилиндрической границы опорного валка задавался запрет перемещений по вер- тикали и в направлении продольной оси бруса.

На верхней половине цилиндрической границы нагружающего валка задавались кинематические граничные условия в виде предписанных вертикальных перемещений. В областях контактного взаимодействия между бруском и валками генерировался контактный интерфейс. В качестве алгоритма обеспечения контакта на интерфейсе использовался расширенный метод множителей Лагранжа.

Решение в нелинейной постановке производилось для серии шагов - возрастающих значений предписанных перемещений с поиском равновесного состояния на каждом шаге алгоритмом Ньютона-Рафсона и обращением матрицы жёсткости методом Холецкого.

В качестве результатов определяли нагрузку на образце в виде реакции в опорах и вертикальные перемещения в шести точках внешней боковой поверхности образца (рис. 1): по три точки в сечениях под нагружающим и над опорными валками. В каждом сечении точки располагались в месте контакта валка и образца (точки 1), в середине сечения (точки 2) и на максимальном удалении от контактной зоны (точки 3). Выбор боковой поверхности для измерений обусловлен тем, что в реальном эксперименте именно эта поверхность подвергается наблюдению.

Для восстановления модуля упругости определялся расчетный прогиб образца $\Delta_{3}$ на ступень его нагружения равный разности между перемещениями соответствующих точек под нагружающим валком $\Delta_{1}$ и над опорным валком $\Delta_{2}$. Удаленные от места контакта точки 3 наименее подвержены влиянию локальных особенностей, возникающих в контактной зоне. Для пересчёта указанных комбинаций в модуль упругости использовалась известная формула для прогиба при трёхточечном изгибе:

$$
E=\frac{\Delta P l^{3}}{48 \Delta_{3} I_{z}},
$$

где $\Delta P-$ шаг нагрузки (ступень нагружения образца), $l$ - расстояние между опорными валками, $I_{z}=b h^{3} / 12-$ осевой момент инерции сечения образца, $b$ и $h-$ соответственно ширина и высота сечения образца. В качестве шага по нагрузке бралась ступень $0-100 \mathrm{H}$, а в качестве прогибов - комбинации приращения перемещений, соответствующих данным ступеням нагружения. В Табл. 1 приведены значения перемещений, опре-

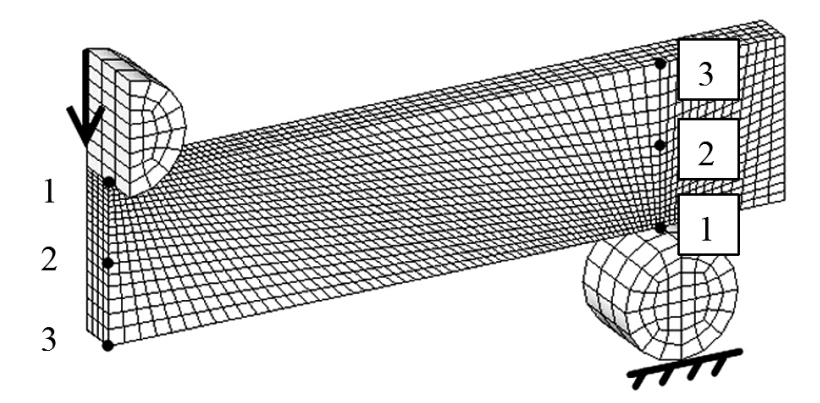

Рис. 1. Расчетная схема модели образца при трехточечном изгибе.

Fig. 1. A design scheme of model of the sample under three-point bending. 
делённые на внешней боковой поверхности в точках 1, 2, 3 в графитовых и стальных образцах на шаг нагрузки 100 Н при $l / h=7$.

Восстановленные значения модулей упругости для всех комбинаций отношений $l / h$ приведены в Табл. 2.

Расчет показал, что по мере увеличения отношения $l / h$ снижается зависимость результата от выбора конкретной комбинации перемещений для расчёта. Так, при величине $l / h=7$, отклонение от истинного значения $E$ составляет не более $2 \%$, а при $l / h=10-$ не более $1,5 \%$, при чем в большую сторону. При малых значениях $l / h$ выбор комбинации играет существенную роль при выборе «невыгодных» комбинаций ошибка достигает $10-20 \%$. Наиболее близкий к истинному результат, в таком случае, даёт комбинация перемещений по противолежащим точкам. Кроме того, величина восстановленного модуля практически не зависит от применяемого шага по нагрузке, что свидетельствует о линейной зависимости всех комбинаций перемещений от приложенной силы.

Основной причиной расхождений при малых отношениях $l / h$ представляется, естественно, несоответствие

Табл. 1. Перемещения точек 1, 2, 3 на поверхности графитовых и стальных образцов на шаг нагрузки $100 \mathrm{H}$ при $l / h=7$.

Table 1. Displacements the points 1, 2, 3 on the surface of a graphite and steel samples, for a load step of $100 \mathrm{~N}$ at $l / h=7$.

\begin{tabular}{|c|c|c|c|}
\hline $\begin{array}{c}\text { Точки измерения } \\
\text { Measuring point }\end{array}$ & $\Delta_{1}, \mu \mathrm{m}$ & $\Delta_{2}, \mu \mathrm{m}$ & $\Delta_{3}, \mu \mathrm{m}$ \\
\hline \multicolumn{4}{|c|}{ Графит МПГ-6 / Graphite MPG-6 } \\
\hline 1 & 118,99 & 5,43 & 113,56 \\
\hline 2 & 118,49 & 7,16 & 111,33 \\
\hline 3 & 117,92 & 7,38 & 110,54 \\
\hline \multicolumn{5}{|c|}{ Сталь Ст.3 / Steel St.3 } \\
\hline 1 & 14,30 & 1,09 & 13,21 \\
\hline 2 & 14,03 & 1,31 & 12,72 \\
\hline 3 & 13,87 & 1,31 & 12,56 \\
\hline
\end{tabular}

Табл. 2. Восстановленные значения модулей упругости $E$, ГПа по вычисленным перемещениям точек (Табл. 1).

Table 2. The restored values of the modulus of elasticity $E$, GPA calculated for displacements of the points (Table 1).

\begin{tabular}{|c|c|c|c|}
\hline $\begin{array}{c}\text { Точки измерения } \\
\text { Measuring роint }\end{array}$ & $l / h=4$ & $l / h=7$ & $l / h=10$ \\
\hline \multicolumn{4}{|c|}{ Графит МПГ-6/ Graphite MPG-6 } \\
\hline 1 & 8,4 & 10,8 & 11,0 \\
\hline 2 & 10,4 & 11,0 & 11,1 \\
\hline 3 & 10,7 & 11,1 & 11,1 \\
\hline \multicolumn{4}{|c|}{ Сталь Ст.3 / Steel St.3 } \\
\hline 1 & 143,5 & 185,5 & 195,7 \\
\hline 2 & 171,7 & 192,6 & 198,0 \\
\hline 3 & 180,0 & 195,0 & 199,1 \\
\hline
\end{tabular}

реальной схемы деформирования бруса положениям, введённым балочной теорией. Помимо этого, присутствуют другие особенности задачи, связанные, прежде всего, с неидеальностью объекта исследования и схемы эксперимента. Некоторые из таких особенностей можно выявить, рассмотрев характер распределения перемещений по высоте сечения. В обоих сечениях видна особенность перемещений в контактной зоне. Величина и протяжённость этой особенности слабо зависит от типоразмера испытываемого бруса и определяется только нагрузкой на валке. При нагрузке $100 \mathrm{H}$ величина особенности составляет 0,4 мкм. Перемещения в части сечения, не затронутой контактной особенностью, являются практически постоянными по высоте. Таким образом, заметный вклад дополнительные перемещения, вызванные контактом с нагружающим валком, вносят только в брусках с малым отношением. Так, для бруска с $l / h=4$ средняя по сечению величина перемещений составляет 23,8 мкм, и особенность вносит отклонения порядка $10 \%$.

На боковых сечениях особенность выражена заметно слабее, так как контактная нагрузка на опорных валках составляет половину от нагрузки на нагружающем валке. Линейный характер распределения перемещений по сечению с переходом через ноль на срединной линии свидетельствует о повороте сечения вокруг мнимого шарнира, находящегося на пересечении срединной линии бруса и вертикали, проходящей через центр опоры. Это хорошо согласуется с представлениями о деформировании бруса, описываемыми балочной теорией изгиба [6]. Одновременно, этот факт объясняет наибольшую стабильность результатов при использовании комбинаций по точкам на срединной линии.

\section{3. Методика испытания образцов}

Образцы изготовлены из листовой стали толщиной 3,5 мм и графита толщиной 7 мм. Механические испытания образцов на растяжение по ГОСТ 1497 - 84 и трехточечный изгиб проведены на испытательных машинах Instron 5982 и Instron 5966 с предельными нагрузками, соответственно, 100 и 10 кН. В Табл. 3 приведены механические свойства и модули Юнга материалов, из которых изготовлены изгибные образцы.

В процессе нагружения образцов на трехточечный изгиб регистрировали диаграммы нагрузка $P$ - перемещение траверсы испытательной машины $f$ и одновременно использовали метод цифровой корреляции изо-

Табл. 3. Механические свойства и модули Юнга испытанных материалов.

Table 3. Mechanical properties and Young's modules of the tested materials.

\begin{tabular}{|c|c|c|c|}
\hline $\begin{array}{c}\text { Материал } \\
\text { Material }\end{array}$ & $\begin{array}{c}\sigma_{\text {т, }}, \mathrm{M \Pi а} \\
\sigma_{\mathrm{y}}, \mathrm{MPa}\end{array}$ & $\begin{array}{c}\sigma_{\text {в }}, \mathrm{M \Pi a} \\
\sigma_{\mathrm{s}}, \mathrm{MPa}\end{array}$ & $\begin{array}{c}E, \text { ГПа } \\
E, \mathrm{GPa}\end{array}$ \\
\hline $\begin{array}{c}\text { Сталь Ст.3 } \\
\text { Steel St.3 }\end{array}$ & 254 & 355 & 200 \\
\hline $\begin{array}{c}\text { Графит MПГ-6 } \\
\text { Graphite MPG-6 }\end{array}$ & - & 47 & 11,0 \\
\hline
\end{tabular}


бражений (ЦКИ) для определения с высокой точностью перемещений точек образца [7]. С помощью установки Vic-3DRT регистрировали смещения элементов системы хаотически распределенных по поверхности объекта и неразрывно связанных с ним малоразмерных метокпятен - спекл-структуры.

На рис. 2 показаны цветная картина поля прогиба и значения в характерных точках, зарегистрированные на боковой поверхности графитового образца с соотношением $l / h=4$ на ступень нагрузки $100 \mathrm{H}$.

Первоначальной задачей было экспериментальное определение областей упругого деформирования образцов по диаграммам $P$-f. Диаграмма прогиба образца из графита представляет собой линейную зависимость вплоть до момента разрушения образца. По диаграмме прогиба стального образца фиксировали нагрузку начала текучести $P_{T}$ и по ней - предел текучести стали при изгибе по формуле (2)

$$
\sigma_{T}=\frac{6 P_{T} l}{4 b h^{2}},
$$

По нагрузке разрушения образца из графита определяли предел прочности графита при изгибе. В Табл. 4 приведены результаты испытания на изгиб образцов из стали и графита при различном соотношении $l / h$.

Как и следовало ожидать, определенные при изгибе образцов трех типоразмеров предел текучести стали и предел прочности графита не зависят от отношения

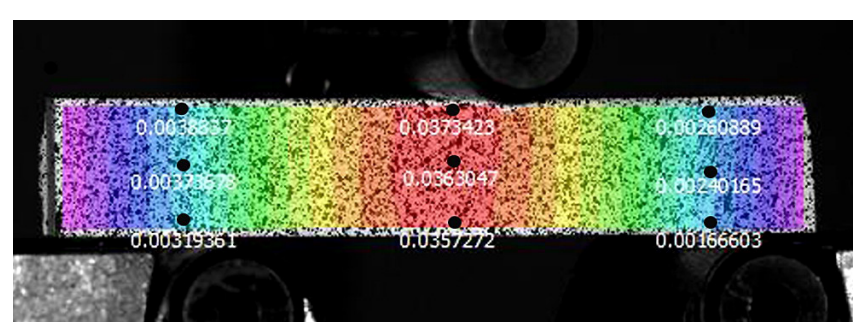

Puc. 2. (Color online) Цветная картина поля прогиба и значения в характерных точках, (мм).

Fig. 2. (Color online) The color pattern field of the deflection and values at characteristic points, $(\mathrm{mm})$.

Табл. 4. Результаты испытания образцов из стали и графита на изгиб.

Table 4. The test results of specimens from steel and graphite for bending.

\begin{tabular}{|c|c|c|c|c|}
\hline & \multicolumn{2}{|c|}{$\begin{array}{c}\text { Сталь Ст.3 } \\
\text { Steel St.3 }\end{array}$} & \multicolumn{2}{|c|}{$\begin{array}{l}\text { Графит МПГ-6 } \\
\text { Graphite MPG-6 }\end{array}$} \\
\hline$l / h$ & $\begin{array}{l}P_{T}, \mathrm{KH} \\
P_{\mathrm{y}}, \mathrm{kN}\end{array}$ & $\begin{array}{l}\sigma_{\mathrm{T}}^{\mathrm{u}}, \mathrm{MПа} \\
\sigma_{\mathrm{y}}^{\mathrm{b}}, \mathrm{MPa}\end{array}$ & $\begin{array}{l}P_{\text {макс }}, \mathrm{\kappa} H \\
P_{\max }, \mathrm{\kappa N}\end{array}$ & $\begin{array}{c}\sigma_{\mathrm{s}}^{\text {и }}, \mathrm{M \Pi а} \\
\sigma_{\mathrm{s}}^{\mathrm{b}}, \mathrm{MPa}\end{array}$ \\
\hline 4 & 1,260 & 308,6 & 1,05 & 64,3 \\
\hline 7 & 0,670 & 287,1 & 0,703 & 75,3 \\
\hline 10 & 0,478 & 292,6 & 0,446 & 68,2 \\
\hline \multicolumn{2}{|c|}{$\begin{array}{c}\text { Средние значения } \\
\text { Average values }\end{array}$} & 296,1 & & 69,3 \\
\hline
\end{tabular}

$l / h$ и равны соответственно 296,1 и 69,3 МПа. Эти результаты хорошо согласуются с известными данными о превышении на 20\% предела текучести пластичной стали при изгибе над пределом текучести при растяжении [8] и примерно двукратным превышением предела прочности графита при изгибе над пределом прочности при растяжении [9].

На линейных участках диаграмм прогиба образцов выявляли диапазон нагрузок, который использован при ступенчатом их нагружении. Одновременно производилась регистрация полей вертикальных перемещений на боковой поверхности образов методом ЦКИ с помощью установки Vic-3DRT.

Предварительные исследования выявили смятие материала образца в месте контакта нагрузочного и опорных валков, причем, тем большее, чем меньше расстояние между опорами. Таким образом, общий прогиб образца $f$, фиксируемый диаграммным аппаратом испытательной машины, складывается из собственно прогиба образца, суммарного смятия материала у опор и нагружающего валка и податливости испытательной машины. Чтобы выделить собственно прогиб образца достаточно вычесть из измеренного перемещения точек в сечении под нагружающим валком усредненное перемещение соответствующих точек в сечении над опорными валками. Такие измерения были проведены для всех типоразмеров образцов двух испытанных материалов.

Модуль Юнга E материала балки подсчитывали по формуле (1). Расчетный прогиб образца $\Delta_{3}$ определяли как разность перемещения точек образца под нагружающим валком $\Delta_{1}$ и усредненным перемещением точек над опорами $\Delta_{2}$.

\section{4. Результаты исследования}

В Табл. 5 приведен пример определенных методом ЦКИ перемещений точек боковой поверхности образца из графита на шаг нагрузки $100 \mathrm{H}$ и расчетные значения соответствующих им модулей упругости Е для отношения $l / h=7$.

Использование перемещения траверсы испытательной машины, даже с учетом ее податливости, приводит к еще большей ошибке в определении модуля упругости материала $-8,5$ ГПа при $\Delta=145$ мкм для графита и 138,1 ГПа при $\Delta=18$ мкм для стали.

Полученные результаты измерений показывают, что значения перемещений различных точек сечений под нагружающим валком и над опорными валками остаются практически постоянными и зависят только от отношения $l / h$. Этот результат качественно хорошо согласуется с результатами расчетного анализа, приведенными в Табл. 1, но количественно отличается. Перемещения точек реальных образцов больше значений, приходящихся на $100 \mathrm{H}$ расчетной нагрузки, примерно на $25 \%$.

В Табл. 6 приведены восстановленные значения модулей упругости для стали и графита, полученные для различных отношений $l / h$.

Их сравнение с результатами расчетного анализа 
также показывает хорошее качественное соответствие, но значительное количественное расхождение. Наилучшее соответствие экспериментально определенных по формуле (1) значений модуля упругости с действительным (расхождение не превышает 1\%) получено для отношений $l / h=10$ независимо от места измерения перемещений. Наибольшее расхождение, превышающее $30 \%$, получено для отношения $l / h=4$, при учете перемещений точек, примыкающих к месту контакта нагружающего и опорных валков. Для отношения $l / h=7$ ошибка определения модуля упругости может составлять 3,5 и $15 \%$ для стали и графита соответственно.

\section{5. Выводы}

Таким образом, по результатам выполненного исследования можно сделать следующие выводы:

Табл. 5. Значения перемещений в точках поверхности образцов из графита и стали на шаг нагрузки $100 \mathrm{H}$ и расчетные значения соответствующих им модулей упругости $E$ для отношения $l / h=7$.

Table 5. The displacement values at the points on the surface of the sample of graphite, for a load step of $100 \mathrm{~N}$ and calculated values of their corresponding moduli of elasticity $E$ for the relation $l / h=7$.

\begin{tabular}{|c|c|c|c|c|}
\hline $\begin{array}{c}\text { Точки измерения } \\
\text { Measuring point }\end{array}$ & $\Delta_{1}, \mu \mathrm{m}$ & $\Delta_{2}, \mu \mathrm{m}$ & $\Delta_{3}, \mu \mathrm{m}$ & $E, \mathrm{GPa}$ \\
\hline \multicolumn{5}{|c|}{ Графит МПГ-6/ Graphite MPG-6 } \\
\hline 1 & 138,6 & 7,1 & 131,5 & 9,3 \\
\hline 2 & 135,1 & 5,7 & 129,4 & 9,5 \\
\hline 3 & 133,2 & 8,2 & 125,0 & 9,8 \\
\hline \multicolumn{5}{|c}{ Сталь Ст.3 / Steel St.3 } \\
\hline 1 & 14,30 & 1,33 & 12,97 & 189,0 \\
\hline 2 & 13,89 & 1,1 & 12,78 & 192,5 \\
\hline 3 & 13,79 & 1,29 & 12,50 & 195,6 \\
\hline
\end{tabular}

Табл. 6. Значения модулей упругости $E$, ГПа.

Table 6. The values of elastic modulus $E$, GPa.

\begin{tabular}{|c|c|c|c|c|c|}
\hline \multirow{2}{*}{$\begin{array}{l}\text { Материал } \\
\text { Material }\end{array}$} & \multirow{2}{*}{$l / h$} & \multicolumn{4}{|c|}{$\begin{array}{c}\text { С учетом перемещений точек } \\
\text { With the move points }\end{array}$} \\
\hline & & $\begin{array}{l}\text { машина } \\
\text { machine }\end{array}$ & 1 & 2 & 3 \\
\hline \multirow{3}{*}{$\begin{array}{l}\text { Сталь Ст.3 } \\
\text { Steel St.3 }\end{array}$} & 4 & 65,5 & 130,6 & 151,7 & 157,7 \\
\hline & 7 & 138,1 & 184,2 & 189,6 & 193,2 \\
\hline & 10 & 162,3 & 193,0 & 197,3 & 198,5 \\
\hline \multirow{3}{*}{$\begin{array}{l}\text { Графит МПГ-6 } \\
\text { Graphite MPG-6 }\end{array}$} & 4 & 6,4 & 7,1 & 7,4 & 7,5 \\
\hline & 7 & 8,5 & 9,3 & 9,5 & 9,8 \\
\hline & 10 & 10,1 & 10,7 & 10,8 & 10,9 \\
\hline
\end{tabular}

1. Применение метода цифровой корреляции изображений при нагружении образцов позволило с большой точностью измерить перемещения поверхностных точек образца и, на их основе, определить фактические величины прогибов балки, используемых при вычислении восстановленных значений модуля упругости материала образца.

2. Значения модуля упругости, определенные при испытании образцов с отношениями $l / h=10$ практически совпадают со средними табличными значениями для исследованных материалов. При соотношении $l / h=10$ не имеет принципиального значения, в каких точках сечения производится измерение перемещений (ошибка измерения находится в пределах 1-3\%), что говорит о малом влиянии смятия материала на смещение точек сечений над опорами.

3. Ошибка при определении значений модуля упругости может составлять 3,5 и $15 \%$ для стальных и графитовых образцов, соответственно, если при испытании использовано соотношение $l / h=7$, и 21 и $32 \%$, если при испытании использовано соотношение $l / h=4$.

4. Использование перемещения траверсы испытательной машины, в качестве величины прогиба балки, некорректно и дает существенно заниженное значение модуля упругости материала.

Благодарность/Acknowledgements. Статья написана в рамках программы по повышению конкурентоспособности НИЯУ «МИФИ».

\section{Литература/References}

1. Yu.G. Dragunov, S.V. Evropin. Atomic energy, 3, 145 - 155 (2015).

2. L. V. Sergeeva. Atomic energy. 3, $157-160$ (2008).

3. A.M. Dmitriev, O.Y. Kavun, M.G. Masenko. Atomic energy. 5, 273-279 (2011).

4. Release 16.2 Documentation for ANSYS [electronic document]/ANSYS Inc. Electronic data and software (104019 files: 10660130531 bytes).

5. NAFEMS search engineering analysis and simulation FEA, Finite Element Analysis, CFD, Computational Fluid Dynamics, and Simulation. NAFEMS Ltd., Hamilton, United Kingdom. (2016).

6. N.M. Belyaev. Mechanics of materials. M.: «Nauka». 1965. 856 p. (in Russian) [Н. М. Беляев. Сопротивление материалов. М.: «Наука». 1965. 856 с.]

7. M. A. Sutton, J. J. Orteu, H. W. Schreier. Image Correlation for Shape, Motion and Deformation Measurements: basic concepts, theory and applications. Springer. P.321. (2009).

8. Y. V. Goltsev. Mechanical testing methods and mechanical properties of materials. Textbook. Moscow: MEPhI, 2012. 228 p. (in Russian) [В. Ю. Гольцев Методы механических испытаний и механические свойства материалов. Учебное пособие. М.: МИФИ, 2012. 228 с.]

9. E.N. Marmer. Carbon and graphite materials. M.: «Metallurgy». 1973. 135 p. (in Russian) [Э.Н. Мармер Углеграфитовые материалы. М.: «Металлургия». 1973. 135 c.] 